\title{
LA RADIOPROTECTION
}

\author{
Editorial
}

\section{Radioprotection et faibles doses}

\section{par le Docteur Jean-Claude Nénot, Institut de Protection et de Sûreté Nucléaire}

\author{
“Nous n'avons ni vrai ni bien qu'en partie, \\ et mêlé de mal et de faux"
}

Pascal

\section{Introduction}

Les connaissances sur les effets biologiques des rayonnements ionisants remontent maintenant à un siècle, puisque les premières constatations de leurs effets délétères ont suivi de très peu la découverte des rayons $X$ par Röntgen en 1895 et celle de la radioactivité par Becquerel en 1896. Les dommages causés par les fortes doses ont été rapidement explorés, en raison des effets aigus qui sont apparus chez certains des premiers utilisateurs, le plus souvent exposés sans protection convenable. Aucun doute n'existe sur la relation de causalité entre les rayonnements et ces effets qui apparaissent dès que suffisamment de cellules sont détruites par les rayonnements. Pour un effet dans un organe donné, il existe un seuil de dose de rayonnements au-dessous duquel le dommage n'est pas détectable ; au-delà de ce seuil, la gravité augmente en rapport avec le nombre de cellules détruites. Les conséquences des doses inférieures à ces différents seuils ont commencé à être étudiées plus tardivement. C'est en 1928 que des médecins réunis à Stockholm à l'occasion du deuxième Congrès International de Radiologie s'inquiétèrent du nombre anormalement élevé de leucémies qui décimaient les pionniers de l'utilisation médicale des rayonnements. C'est d'ailleurs à cette occasion que fût créée la Commission Internationale de Protection Radiologique (CIPR). Les effets tardifs résultent de dommages qui peuvent apparaître dans les cellules de l'individu ou dans ses cellules germinales; dans le premier cas, les dommages peuvent se traduire par l'induction d'un cancer (tumeur solide ou leucémie), dans le second par des troubles héréditaires transmis par la personne exposée à sa descendance. Ces effets semblent apparaître au hasard chez les individus exposés sans qu'il soit possible de prédire lesquels d'entre eux développeront l'effet. C'est en raison de leur caractère aléatoire que ces effets sont dénommés stochastiques. Le risque, c'est-à-dire la probabilité d'occurrence d'une tumeur maligne ou d'une mutation transmise à la descendance dépend de la quantité de rayonnements reçue par l'individu. La notion de dose a été développée pour exprimer ce risque : la dose est l'index du risque. Les connaissances sur ce type d'effets se sont grandement affinées au cours des dernières décennies, notamment en ce qui concerne les phénomènes qui gouvernent l'induction des cancers, les relations de cause à effet qui lient la dose et la probabilité pour un individu exposé de développer la tumeur, les nocivités relatives des différents types de rayonnements, ou encore l'atténuation du risque quand la dose est étalée dans le temps.

Il subsiste cependant un certain nombre de zones d'ombre, particulièrement dans le domaine des faibles doses. L'expression "faible dose " peut être prise dans des sens très variés. Elle peut être tout simplement opposée à " forte dose ", et qualifierait alors toute dose incapable d'induire un effet aigu. Elle peut aussi s'appliquer uniquement aux doses pour lesquelles il est impossible de mettre en évidence un quelconque effet. Cette définition est souvent évoquée et correspond aux doses au-dessous desquelles les études épidémiologiques de populations exposées ne sont plus exploitables à cause de leur puissance statistique insuffisante. Dans une troisième démarche, plus pragmatique, les faibles doses correspondent aux expositions normalement reçues par la population et par les professionnels. Cette définition demeure cependant incomplète en l'absence d'un paramètre essentiel : le débit de dose. Les rayonnements se comportent comme la plupart des autres toxiques : à exposition égale, la nocivité est moindre quand l'agent nocif est délivré sur des périodes longues. Le paramètre temps est au centre de l'évaluation du risque des faibles doses, puisqu'elle repose principalement sur l'extrapolation du risque constaté à des doses relativement élevées reçues pendant des temps très courts. C'est notamment le cas des survivants des bombardements d'Hiroshima et Nagasaki, qui constituent la source principale d'information sur les effets tardifs des rayonnements sur l'homme.

Plusieurs questions, dont les réponses sont malheureusement assorties d'un degré d'imprécision, font aujourd'hui l'objet de débats qui dépassent souvent le cadre scientifique :

1 - peut-on quantifier de façon raisonnable le risque aux faibles doses?

2 - quelle est la forme de la relation entre la dose et le risque?

3 - existe-t-il un seuil de dose au-dessous duquel l'effet n'existe plus?

4 - l'effet s'atténue-t-il avec le temps pour devenir nul ou négligeable au bout de quelques années ou décennies?

5 - existe-t-il des individus particulièrement sensibles (ou particulièrement résistants) aux rayonnements ?

6 - existe-t-il un phénomène biologique d'adaptation aux rayonnements, et les faibles doses auraient-elles des effets bénéfiques, qui pourraient l'emporter sur les effets néfastes? et enfin

7 - la résolution de ces questions seraitelle profitable à la protection contre les rayonnements ou à d'autres disciplines et dans quel sens?

Il existe donc de nombreuses questions, dont l'intrication complique l'interprétation des données.

\section{La quantification du risque aux faibles doses et faibles débits}

La prévision de la probabilité d'apparition de cancers radioinduits ou d'effets héréditaires comporte un passage obligatoire : l'observation de groupes de personnes aussi nombreuses et homogènes que possible, soumises à des doses connues de rayonnements. Les expériences sur 
des animaux de laboratoire et sur des systèmes cellulaires permettent d'étudier les mécanismes de l'induction des cancers radioinduits, mais ne peuvent pas apprécier de façon quantitative le risque chez l'homme. Quelle que soit la méthode utilisée pour le suivi épidémiologique, soit enquête de cohorte qui consiste à suivre un groupe d'individus indemnes de la maladie pendant un temps très long (idéalement jusqu'à l'extinction du dernier individu du groupe), soit étude castémoin qui rassemble des individus présentant la maladie à étudier et parmi lesquels on recherche l'influence de l'agent nocif, la quantification du risque devient très imprécise voire impossible dès que l'agent supposé causal est en faibles quantités. Un problème insoluble est que ces groupes sont soumis à d'autres expositions nocives (souvent difficiles à apprécier), et parfois beaucoup plus importantes ou au mieux du même ordre de grandeur que l'exposition étudiée ; c'est notamment le cas de l'irradiation naturelle.

Les études épidémiologiques assorties d'une puissance statistique suffisante concernent des populations qui ont subi des irradiations aiguës à doses relativement élevées ; pour apprécier le risque aux faibles doses reçues à de faibles débits, il faut donc procéder à des extrapolations qui sont nécessairement assorties d'incertitudes. Le Comité scientifique des Nations Unies (United Scientific Committee on the Effects of Atomic Radiation, couramment dénommé par ses initiales UNSCEAR) estime que le risque aux faibles doses délivrées à faibles débits est entre 2 et 10 fois plus petit que le risque aux fortes doses. Cette valeur de la réduction de l'efficacité des rayonnements aux faibles doses a été admise par l'Académie des sciences américaine en 1990. L'année suivante, pour des raisons de facilité d'utilisation et à titre conservatif, la CIPR n'a retenu qu'une seule valeur, la plus basse. Les études de radiobiologie fondamentale, peuvent fournir, dans le domaine des faibles doses, des données intéressantes pour évaluer la réduction du risque avec la diminution du débit.

Le risque lié à l'exposition aux rayonnements dure pendant des décennies, voire pendant toute la vie de l'individu. Pour évaluer le risque pendant la vie entière des individus il faut donc s'appuyer sur des modèles de projection, puisque les populations observées sont loin d'être éteintes. Deux modèles simples sont en général utilisés : le modèle additif qui suppose que l'excès de risque demeure constant avec le temps, indépendamment du taux naturel de cancers et le modèle multiplicatif dans lequel l'excès lui est proportionnel. Le modèle multiplicatif aboutit à un risque supérieur à celui obtenu par le modèle additif, puisque le taux naturel des cancers croît avec l'âge. L'UNSCEAR estime depuis 1988 que le modèle multiplicatif fournit une évaluation correcte du risque de cancer radioinduit. L'UNSCEAR reconnaît qu'il est possible que le modèle multiplicatif, s'il donne une appréciation acceptable du risque moyen dans une population, puisse conduire à sa surestimation s'il existe une diminution du risque avec l'âge.

Le facteur de réduction, qui permet de passer du risque aux fortes doses et forts débits au risque aux faibles doses et faibles débits, alimente un débat contradictoire. L'Académie des Sciences française a estimé en octobre 1995 que ce facteur est sous-estimé par l'UNSCEAR et la CIPR ; il pourrait exister une discontinuité dans le mécanisme de réparation de l'ADN, selon que la dose, cause du dommage initial, a été forte ou faible 1 . En effet, les faibles doses ne génèrent que peu de réparations fautives, à l'inverse des fortes doses. En plus, un mécanisme de prolifération des cellules survivantes contribue à atténuer le dommage et s'ajoute au mécanisme de réparation. Le débat tourne en fait autour de la forme de la relation doseeffet aux faibles doses : le facteur de réduction est-il dépendant de la dose et du débit? En d'autres termes, la relation linéaire-quadratique, qui est admise pour exprimer la variation du risque avec la dose, est-elle réellement linéaire aux faibles doses et sa pente ne surestime-telle pas le risque?

\section{La relation entre la dose et l'effet}

En 1962, I'UNSCEAR a estimé qu'une relation dose-effet linéaire (et sans seuil) pouvait donner une image réaliste des phénomènes de la radiocancérogenèse. Compte tenu de la diversité des paramètres physiques liés aux rayonnements, de la grande disparité qui existe entre les organes et systèmes qui régentent les phénomènes de la vie et de sa régulation, et surtout de la complexité des phénomènes biologiques mis en jeu lors des différentes étapes de la radiocancérogenèse, on serait tenté de penser qu'il n'existe pas de relation universelle qui traduise l'ensemble des effets stochastiques. II existe cependant nombre d'arguments scientifiques qui plaident pour une relation linéaire aux faibles doses, assortie de pentes spécifiques à l'effet considéré. Parmi ces arguments figure au premier plan l'hypothèse de l'origine monoclonale de la plupart des cancers cette thèse, défendue par la plupart des experts et rarement rejetée de façon catégorique par les autres, soutient qu'une lésion unique dans une seule cellule constitue la première étape indispensable au développement du cancer. II est difficile d'imaginer une cellule isolée au sein de l'organisme sans interaction avec son environnement et d'ignorer ainsi la contribution d'autres cellules. A ce propos, il convient de souligner que les phénomènes biologiques et les étapes du développement d'un cancer radioinduit sont extraordinairement complexes et qu'une relation simple ne constitue qu'une représentation schématique.

Depuis qu'elle a été proposée, la relation linéaire retenue par I'UNSCEAR a été adoptée sans état d'âme excessif par les praticiens de la radioprotection. En effet, parmi les impératifs que doivent s'imposer ces techniciens figure au premier plan l'assurance d'être prudent, en raison des doutes ścientifiques qui subsistent. La relation linéaire répond à cette exigence, puisqu'elle ne sous-estime pas le risque et ne peut que le surestimer, si le facteur de réduction du risque actuellement recommandé pour l'extrapolation aux très faibles doses est trop petit. En outre, cette relation constitue un outil de gestion commode, qui permet une représentation quantifiée du risque et notamment de montrer que le risque lié aux limites de dose est tolérable. La dose collective constitue un outil supplémentaire, qu'autorise la relation linéaire ; elle permet notamment la comparaison de différentes options, dans la mesure où les risques individuels ne sont pas trop disparates. Mais cette commodité ne doit pas faire oublier qu'il ne s'agit que d'un modèle et non d'une description directe de la réalité.

\section{Le seuil}

La plupart des études épidémiologiques n'ont pas une puissance statistique suffisante pour mettre en évidence l'effet des rayonnements au-dessous de doses de l'ordre de 100 à 200 mSv (reçus par un individu pendant toute sa vie). Les derniers résultats de l'étude des survivants d'Hiroshima et Nagasaki entre 1950 et 1990 permettent de fixer à 50 mSv la dose au-dessous de laquelle l'effet n'est plus significatif. Pour une personne du public, ces valeurs sont du même ordre de grandeur que son exposition à l'irradiation naturelle pendant toute sa vie ; pour un travailleur, elles correspondent à moins d'une dizaine d'années d'exposition à la limite de $20 \mathrm{mSv}$ actuellement recomman-
(1) L'ADN, ou acide désoxyribonucléique, est le constituant primordial des chromosomes des noyaux cellulaires qui porte l'information génétique de la cellule vers la génération suivante, lors de la division cellulaire L'effet des rayonnements est dô aux gésération suvante, lors de la division cellulaire. L'efet des rayonnemest du aux systèmes de réparation plus ou moins rapides et fidèles. Ainsi, dans le cas de lésion sur un seul brin de l'ADN (cassure simple-brin) la réparation est rapide et s'effectue sans erreur, le brin indemne servant de modèle. La réparation est beaucoup plus difficile quand les deux brins sont lésés (cassure double-brin) et est sujette à l'erreur. 
dée. C'est pourquoi, à ces niveaux de doses, il est tentant d'imaginer un " seuil pratique " ; ce seuil, qui se confond avec le seuil de détection des effets, pourrait aussi être lié aux grands temps de latence des cancers induits par des faibles doses, qui ne permettraient pas leur expression avant la fin de la vie de l'individu.

II existe des arguments scientifiques en faveur du seuil, qui reposent sur les mécanismes de la radiocancérogenèse. Quelque 5.000 à 10.000 dommages surviennent par heure dans l'ADN, liés à des agressions de tous ordres. Les phénomènes de réparation peuvent empêcher l'expression de ces dommages. II est donc possible de soutenir, tout au moins au niveau de l'ADN, qu'un petit incrément de dose, responsable de lésions ponctuelles en nombre très réduit, peut être considéré comme négligeable pour le risque. En fait cette thèse passe sous silence d'autres lésions causées par les rayonnements à l'ADN, beaucoup plus rares que les précédentes, dites " cassures double-brin ", mais dont la réparation est plus délicate. Ces dernières lésions sont en faveur de la thèse d'une augmentation progressive du risque avec l'augmentation progressive de la dose.

La preuve de l'existence ou de l'absence d'un seuil de dose et de débit de dose pour l'induction des cancers radioinduits ne pourra être apportée par les seules études épidémiologiques. L'UNSCEAR estime que les études épidémiologiques sont incapables de déceler des augmentations de risque (par rapport au risque naturel de cancer) inférieures à 20-30 \%, mais permettent de fixer les limites supérieures du risque. II commence à être possible d'identifier les cancers radioinduits parmi les cancers dits naturels, grâce à une " signature " spécifique laissée par les rayonnements au niveau moléculaire. Le développement généralisé de cette technique augmenterait dans de grandes proportions la puissance des études épidémiologiques. Cependant, étant donné la très grande variété des cancers des différents organes, cette cartographie ne sera pas complétée avant de nombreuses années. L'identification de cette signature aurait des conséquences dans d'autres domaines, comme celui de l'indemnisation des cancers professionnels, dont l'origine serait alors fermement établie.

S'il s'avère que les processus de réparation cellulaire à faibles doses contrebalancent les dommages causés par les rayonnements, on peut imaginer qu'il est nécessaire de réviser le système actuel de protection. II conviendrait alors de tenir compte de plusieurs paramètres, dont les principaux sont :

1 - la valeur quantitative des seuils, liée aux organes, aux doses et aux débits :
2 - la rationalité du choix de ces seuils ;

3 - les nocivités des divers types de rayonnements. En se basant sur les seuls résultats de l'épidémiologie, un seuil autour de 100 mSv (dose vie) paraîtrait alors raisonnable. Le degré d'incertitude autoriserait facilement un facteur 2 audessus et au-dessous de cette valeur, selon que l'on est plus ou moins prudent. L'ensemble du débat est évolutif ; la solution, qui est entre les mains de la radiobiologie fondamentale, n'existe malheureusement pas à l'heure actuelle.

Un paramètre supplémentaire non négligeable doit être pris en compte ; c'est la radioactivité naturelle. Les expositions sujettes à limitation réglementaire s'ajoutent à l'exposition naturelle, qui se situe en moyenne entre 100 et 200 mSv au cours d'une vie. Les personnes du public seraient donc exposées pendant leur vie entière à des doses qui sont du même ordre de grandeur que le seuil, en admettant que ce dernier soit au niveau cité plus haut. L'exposition ajoutée du fait des rayonnements d'origine artificielle se situerait donc au-dessus de ce seuil, tout en étant du même ordre de grandeur. Les doses professionnelles, qui sont supérieures à celles du public d'environ un ordre de grandeur, le dépasseraient très nettement. Dans ces conditions, le sys tème de protection qui serait appliqué ne différerait pas fondamentalement de l'actuel, au moins dans le cas des expositions professionnelles.

\section{L'atténuation de l'effet avec le temps}

Les modèles de projection du risque couramment utilisés ne prennent pas en compte le facteur temps. Or il est vraisemblable qu'il existe un certain " gommage " de l'effet au fur et à mesure que l'on s'éloigne du moment de l'irradiation. Ce phénomène est connu depuis longtemps pour les leucémies radioinduites, qui sont plus précoces que l'ensemble des autres cancers et qui présentent un pic trois à dix ans après l'exposition et qui diminuent ensuite lentement. Les autres cancers apparaissent plus tardivement mais augmentent plus graduellement dans le suivi des survivants japonais des bombardements atomiques, l'augmentation, pour un âge et un sexe donnés, est monotone avec le temps et proportionnelle à l'augmentation avec l'âge du fond naturel des cancers. II faudra attendre l'extinction totale de ces personnes pour confirmer ce résultat, qui semble dément par d'autres études dans lesquelles la courbe a tendance à s'infléchir avec le temps. Ce fait est patent pour les personnes qui ont été exposées dans les premières années de leur vie. L'examen de l'ensemble des données actuellement disponibles montre que la prise en compte de la diminution du risque avec l'âge conduirait à diminuer de 20 à $40 \%$ l'estimation courante du risque sur la vie entière.

\section{La sensibilité génétique}

II existe indéniablement des individus plus sensibles aux rayonnements que la moyenne. Cette question n'est pas spécifique aux rayonnements, puisque l'être humain n'est pas égal devant le risque, quel qu'en soit l'agent causal. L'amélioration des connaissances sur le génome humain et sur les capacités de réparation de la cellule permet d'identifier certains individus particulièrement sensibles. Cette identification est certes à ses débuts, mais il ne faut pas ignorer qu'elle pourrait à terme soulever des problèmes délicats, touchant par exemple la politique de l'emploi, puisqu'impliquant une certaine discrimination entre travailleurs, contraire aux règles courantes. De plus, la discrimination sur des bases génétiques est actuellement rejetée par les comités d'éthique nationaux et internationaux qui se sont penchés sur cette question. Le résultat des recherches en cours dira si cette question doit être soulevée dans le domaine de la protection contre les rayonnements. Deux solutions extrêmes seraient également inacceptables : (1) une sélection se ferait à l'embauche pour ne retenir que les individus les moins sensibles et cela pourrait se traduire par une augmentation des limites ; (2) à l'inverse les limites seraient adaptées aux sujets les plus sensibles ; nombre de pratiques utilisant les rayonnements devraient être interdites, puisque quelques rares individus sont dix à cent fois plus sensibles que la moyenne. Le détriment pour la société, qui se priverait des avantages qu'elle tire de l'utilisation des rayonnements, serait considérable. La solution serait vraisemblablement intermédiaire, et consisterait à accepter les inégalités biologiques, dans la mesure où elles n'entraînent pas de détriment inacceptable pour l'individu et la société.

\section{La réponse adaptative, les effets bénéfiques}

II est démontré depuis déjà de nombreuses années que de faibles doses peuvent entraîner des modifications bénéfiques dans les cellules et les organismes vivants, leur conférant une certaine capacité d'adaptation aux rayonnements. Cet effet semble être lié à une augmentation des capacités de réparation de l'ADN. Quels que soient les mécanismes impliqués, ils sembleraient capables d'agir non seulement sur les lésions radioinduites mais aussi sur une partie des lésions induites par d'autres agents toxiques. II a été suggéré qu'il 
pourrait en résulter une stimulation des mécanismes de réparation et de ce fait réduire le taux des cancers naturels ains que celui des cancers radioinduits. Les nombreuses expériences animales et les quelques données humaines disponibles ne permettent pas d'affirmer que la réponse adaptative diminue l'incidence d'effets tardifs tels que l'induction de cancers à faibles doses chez l'homme. La biologie moléculaire devrait permettre d'éclaircir les mécanismes fondamentaux de cette réponse adaptative. D'ici là, il semble prématuré de conclure que ce phénomène est bénéfique et qu'il l'emporte sur les effets néfastes des rayonnements.

\section{Conclusions}

La radiobiologie et la radioprotection ne doivent pas être confondues. La première est une science alors que la seconde est une discipline qui s'appuie sur les données de la première mais qui fait aussi appel à d'autres considérations. C'est aussi une erreur d'opposer les deux méthodes qui permettent d'acquérir les données utiles pour l'appréciation du risque : l'épidémiologie et la biologie fondamentale. Ces méthodes sont complémentaires, et aucun progrès sérieux ne sera fait qui ne sera étayé à la fois par l'observation des effets sur l'homme et l'expérience en laboratoire. Le champ d'application de la radiobiologie dépasse d'ailleurs largement le cadre de la protection, puisque beaucoup de ses résultats contribuent à une meilleure connaissance des mécanismes essentiels de la cancérogenèse
La radioprotection vise à prévenir les risques des rayonnements plutôt qu'à en guérir les effets. Elle s'appuie donc sur trois démarches successives et complémentaires : l'évaluation du risque, la prise de décision et l'application. Dans ce processus itératif, les connaissances et les jugements de valeur jouent des rôles plus ou moins importants. Pour la première démarche, l'évaluation, on peut considérer que la nature du risque et l'ordre de grandeur des phénomènes sont connus de façon convenable ; le jugement de valeur n'intervient que dans le classement en importance des connaissances acquises. La deuxième démarche, la prise de décision, aboutit à l'établissement de règles de conduite, notamment à la fixation des limites réglementaires ; elle ne repose pas uniquement sur les connaissances scientifiques mais aussi sur des paramètres techniques, sociaux et économiques. Comme la part de ces derniers est importante, il est indispensable de disposer d'une référence, sous forme de consensus. C'est le rôle de la CIPR et de grandes organisations internationales comme I'UNSCEAR, I'Agence Internationale de l'Energie Atomique, l'Agence de l'Energie Nucléaire de l'OCDE ou l'Union Européenne, de veiller au maintien d'une harmonisation exemplaire. La dernière démarche, l'application, s'attache à veiller au respect des objectifs de protection et des stratégies définies dans l'étape précédente et la partie technique l'emporte sur les autres. II s'agit, dans cette dernière étape, d'évaluer le niveau d'exposition, la dose n'étant pas mesurable directement; les experts doivent se livrer à des estimations (mesures d'activité, modèles de transfert). II faut aussi s'efforcer d'expliciter les marges de sécurité qui sont introduites et définir de façon réaliste les niveaux d'exposition.

II subsiste encore de nombreuses inconnues ou incertitudes sur les effets des faibles doses. La question-clé pour la radioprotection : est-il possible de gérer convenablement le risque radiologique? concerne l'étape de "prise de décision ", avec ses composantes objectives et subjectives, de poids variable. Le débat est difficile, mais contribue à la progression des connaissances. II est cependant possible de douter de sa réelle utilité, sous le seul angle des implications pratiques pour les années à venir. II s'agit finalement de discuter et choisir entre un risque très faible et un risque nul. Pour le public, dont les expositions sont à des niveaux extrêmement bas, le choix ne devrait pas entraîner de différences notables dans la pratique. Pour les travailleurs, dont les niveaux d'exposition sont d'un ordre de grandeur plus grand que ceux du public, le débat doit être centré sur la réduction des expositions effectivement reçues afin de les amener à un niveau raisonnable ; c'est l'optimisation de la protection, principe fondamental de la radioprotection. Ceci implique le suivi attentif des expositions professionnelles, ainsi que des actions menées aux moments de la conception des installations et des opérations. Même s'il est à l'évidence simplificateur, le système de protection contre les rayonnements restera sans doute stable dans les prochaines années. Son évolution dépendra de l'émergence de connaissances qui feraient l'objet d'un consensus international et qui prouveraient de façon irréfutable qu'elles entraîneraient des progrès significatifs de la gestion du risque radiologique.

\section{Bibliographie}

\section{Académie des Sciences}

Problèmes liés aux effets des faibles doses des radiations ionisantes.

Rapport n³4, octobre 1995, Lavoisier, Paris, (1995)

\section{Birraux C.}

Contrôle de la sûreté et de la sécurité des installations nucléaires. Office parlementaire d'évaluation des choix scientifiques et technologiques. Assemblée Nationale, $n^{\circ} 1825$, Sénat $n^{\circ}$ 172. (1994); Assemblée Nationale $n^{\circ} 2651$, Sénat, $n^{\circ} 278$, (1996)

\section{Comité scientifique des Nations Unies pour l'étude des effets des rayonnements ionisants Sources and effects of ionizing radiation}

UNSCEAR 1994 Report to the General Assembly. United Nations, (1994)

\section{International Commission on Radiological Protection}

1990 Recommendations of the International Commission on Radiological Protection.

ICRP 60, Annals of the ICRP, vol 21, $n^{\circ} 1-3$, Pergamon Press, (1991)

\section{National Academy of Sciences}

Health effects of exposure to low levels of ionizing radiation.

BEIR V Report, National Academy Press, Washington, DC, (1990)

Pierce D.A., Shimizu Y., Preston D.L., Vaeth M. and Mabuchi K.

Studies of the mortality of atomic bomb survivors. Report 12 , Part 1. Cancer: 1950-1990

Radiation Research, 146, 1-27 (1996)

\section{Tubiana M.}

Effets cancérogènes des faibles doses du rayonnement ionisant. Radioprotection, Vol 31, n² 2, avril-juin 1996 (1996). 